\title{
from Eastern Spain
}

3

4 Paula Sánchez-Thevenet ${ }^{1, *}$, David Carmena $^{2,{ }^{*}}$, Manuel Adell-Aledón ${ }^{1}$, Elena Dacal ${ }^{2}$, $5 \quad$ Eva Arias $^{2}$, José María Saugar ${ }^{2}$, Esperanza Rodríguez ${ }^{2}$, María Auxiliadora Dea-

$6 \quad$ Ayuela $^{3}$

$7 \quad{ }^{1}$ Department of Medicine, Cardenal Herrera-CEU University, CEU Universities,

$8 \quad$ Castellón de la Plana, Castellón, Spain

$9 \quad{ }^{2}$ Parasitology Reference and Research Laboratory, National Centre for

10 Microbiology, Health Institute Carlos III, Majadahonda, Madrid, Spain

$11{ }^{3}$ Faculty of Health Sciences, Cardenal Herrera CEU University, CEU Universities, Alfara del Patriarca, Valencia, Spain 


\section{Abstract}

The diversity and frequency of enteric parasites in dog populations in the Castellón province (Eastern Spain) was assessed by means of a prospective crosssectional epidemiological survey. A total of 263 canine faecal samples were collected between July 2014 and July 2016. Detection of intestinal parasites was conducted by routine coprological methods. In addition, identification of Giardia duodenalis and Cryptosporidium spp. was carried out by direct immunofluorescence microscopy, whereas the presence of Strongyloides spp. was assessed by real-time PCR in a selected number of specimens. Based on conventional and/or immunofluorescence microscopy examination, $65.8 \%$ (95\% CI: 59.7\%-71.5\%) of the investigated dogs were found infected by at least one gastrointestinal parasite. Giardia duodenalis (35.4\%) and members of the family Ancylostomatidae (27.0\%) were the most prevalent protozoan and helminth parasites found, respectively. Other pathogens potentially infective to human included Toxocara canis $(8.0 \%)$, Cryptosporidium spp. (6.8\%), and Strongyloides spp. (1.1\%). Frequency of occurrence of helminthic, but not protozoan, enteroparasites was geographical origin-dependent $(P=0.02)$, with dogs living in coastal areas presenting higher infection rates than those living in inland regions. Similarly, rural dogs were significantly more infected than urban dogs $(P<0.001)$. Our results revealed that zoonotic agents were common in dogs from the Castellón province. Animals from rural areas and sheltered dogs were particularly at risk of these infections.

\section{Keywords: Intestinal parasites; Dogs; Prevalence; Zoonoses; Spain.}




\section{Introduction}

51 Intestinal parasites including a wide range of helminth and protozoan species are common infectious agents of dogs. Some of them can be transmitted to humans causing diseases such as hydatidosis by Echinococcus granulosus sensu lato (s.1.), giardiosis by

Giardia duodenalis, and cryptosporidiosis by Cryptosporidium spp. (Soriano et al. 2010; Deplazes et al. 2011; Overgaauw and Knapen 2013; Otranto et al. 2017). transmission harbour greater diversity and higher prevalences (above $80 \%$ ) of parasitic 
an area where the intestinal parasite fauna in the canine host has not been investigated yet.

\section{Material and methods}

\section{Study area and design}

Castellón is a province of the AR of Valencia in Eastern Spain. It has a surface area of over $6,632 \mathrm{~km}^{2}$ and has a total population of 582,327 inhabitants. Most of the population lives in the coastal strip (a third of them in the capital city Castellón de la Plana), whereas the mountainous interior is practically uninhabited. The province is divided in eight administrative regions called "comarcas" (FIG. 1). Agriculture and livestock raising constitute the principal economic activities of the province. The climate of the region is typically Mediterranean, characterized by mild, rainy winters and warm, dry summers.

There were 151,311 domestic dogs officially censed in Castellón in 2015, 21,936 of them belonging to hound-type breeds commonly used in hunting, whereas an undetermined number of guard or shepherd dogs were used in agricultural exploitations in rural areas (Registro Informático Valenciano de Identificación Animal 2015).

Overall. Stray, abandoned, or surrendered animals in the province were managed by five private animal shelters, one of them also acting as a licensed breeding kennel. Surveyed animals were categorised as pet dogs, breeding dogs, sheltered dogs, shepherd dogs and hunting dogs. To achieve the objectives of the present project a cross-sectional study was carried out in this Spanish province between July 2014 and July 2016.

\section{Faecal sample collection}


Estimated sample size $(n=217)$ was calculated using Open Source Epidemiological

Statistics for Public Health OpenEpi 3.01 software (Dean 2013). Power was set considering an expected prevalence of $50 \%$, a marginal error of $7 \%$ with a $95 \%$ confidence interval (CI), and a loss rate of $10 \%$. A total of 263 faecal dropping samples from individual dogs were regularly collected during the study period. Faecal specimens belonged to dogs attended at four of the five animal shelters located at the province $(n=$ 139), breeding dogs for sale $(n=18)$, hunting $(n=68)$, shepherd $(n=24)$ and pet $(n=$ 14) dogs. Faecal specimens were placed in screw-topped specimen containers and uniquely labelled indicating identification number and date of collection. Data on sex, age, status, and geographical origin of the dog and consistency of the faecal material were also recorded.

\section{Parasitological procedures}

Faeces were stored at $4{ }^{\circ} \mathrm{C}$ in $5 \%(\mathrm{v} / \mathrm{v})$ formaldehyde until further treatment.

Macroscopic examination was firstly performed for detection of proglottids and adult worms. After homogenization, each faecal sample was divided into two aliquots. In order to detect parasite eggs, cysts and oocysts, one aliquot was analysed using routine coprological procedures based on the modified Ritchie formalin-ether and Sheather's sugar flotation concentration methods (Thienpont et al. 1979). Each sample was microscopically examined at $10 \times$ in triplicate and suspected parasite structures confirmed at 40× magnification in a Leica DM500 microscope (Wetzlar, Germany). Parasite eggs, cysts and oocysts were identified according to their morphometric characteristics. The second aliquot was used to assess the presence of Giardia duodenalis cysts and Cryptosporidium spp. oocysts by direct fluorescent antibody test (DFAT) using a commercially available kit (MERIFLUOR ${ }^{\circledR}$ Cryptosporidium/Giardia, 
123 Meridian Bioscience, EE.UU). A sample was recorded as positive if at least one parasite 124 form was observed by any given method.

\section{DNA extraction and purification}

127 The presence of Strongyloides spp. was assessed by a PCR-based method (see below) in

128 a limited number of faecal samples from dogs considered at higher risk of being in contact with the nematode, including shepherd and hunting dogs. Aliquots of selected faecal samples were stored in 70\% ethanol. Total DNA was extracted from $200 \mathrm{mg}$ of faecal material using the QIAamp® DNA Stool Mini Kit (Qiagen, Hilden, Germany) following the manufacturer's instructions. Purified DNA samples $(200 \mu \mathrm{L})$ were stored at $-20{ }^{\circ} \mathrm{C}$ and shipped to the Parasitology Reference and Research Laboratory, Spanish National Centre for Microbiology (Majadahonda) for further PCR testing.

\section{Molecular detection of Strongyloides spp.}

Genus-specific (F: 5'-GAATTCCAAGTAAACGTAAGTCATTAGC-3'and R: 5'-

TGCCTCTGGATATTGCTCAGTTC-3') primers were used to amplify a partial sequence of the small subunit ribosomal RNA (ssu rDNA) gene of Strongyloides spp. (Verweij et al. 2009) by a qualitative real-time PCR (qPCR) assay as described elsewhere (Saugar et al. 2015). qPCR reactions $(25 \mu \mathrm{L})$ contained 1× Quantimix venezuelensis L3 was used as positive control. All DNA isolates were assayed in duplicate. An internal inhibition control including $10 \mathrm{ng}$ of $S$. venezuelensis DNA was used for each sample. Negative and no template controls were included in each run. 
148 Cycling conditions were $15 \mathrm{~min}$ at $95^{\circ} \mathrm{C}$ followed by 50 cycles of $10 \mathrm{~s}$ at $95^{\circ} \mathrm{C}, 10 \mathrm{~s}$ at $60{ }^{\circ} \mathrm{C}$ and $30 \mathrm{~s}$ at $72{ }^{\circ} \mathrm{C}$. DNA amplification and detection of fluorescence at the end of each amplification cycle were performed on a Corbett Rotor Gene ${ }^{\mathrm{TM}} 6000$ real-time PCR system (Qiagen). Data were analysed with Rotor Gene 6000 Series software version 1.7.

\section{Data analyses}

Infection rates of total and individual intestinal parasites were determined. The Chisquare $\left(\chi^{2}\right)$ test was used to compare total and individual parasite infection rates in the canine population under study by sex, status, and geographical origin of the animals. Prevalence risk ratios (PRR) with 95\% confidence intervals (CI) were also calculated to assess the association between the above mentioned variables and the occurrence of enteric parasite infections. A probability $(P)$ value $<0.05$ was considered evidence of statistical significance. Data were analysed with the free software RStudio Version 1.0.44 (https://www.rstudio.com/) using the Epitools library.

\section{Results}

The study included dogs from six of the eight administrative regions of the Castellón Province (FIG. 1). Intestinal parasites were found in $65.8 \%(173 / 263 ; 95 \%$ CI: $59.7 \%-$ $71.5 \%$ ) of the faecal samples analysed. Frequencies of appearance of each individual parasite species are shown in Table 1. Helminth $(38.4 \% ; 101 / 263)$ and protozoa $(43.0 \% ; 113 / 263)$ infections were observed in the examined samples with no significant differences $(P>0.05)$. In addition, co-infections involving protozoan and helminth species were detected in $15.6 \%(41 / 263)$ of the samples, whereas no parasite infections were found in $34.2 \%$ 
$173(90 / 263)$ of the faecal specimens examined. Overall, seven species of protozoa, three of 174 cestodes, and seven of nematodes were identified. Intestinal protozoa were the most 175 frequent type of enteric parasites identified in all dog groups excepting shepherd and hunting dogs, which were primarily infected by nematodes (Table 1). The most frequently observed parasite species were Giardia duodenalis (35.4\%), 178 followed by hookworms (27.0\%), Toxocara canis (8.0\%), and Trichuris vulpis $(6.8 \%)$. 179 However, these figures varied largely depending on the dog's status considered (Table 1). Out of the 93 Giardia-positive samples, 90 were detected only by DFAT, whereas the remaining three tested positive both by microscopy examination and DFAT. Additionally, two shepherd dogs carried sporulated Eimeria spp. oocysts. Because this coccidia does not naturally infect dogs this finding very likely reflects events of coprophagy or predation of other species. In samples with a positive result to any given intestinal parasite, single and multiple (two or more) infections were identified in $36.5 \%$ $(96 / 263)$ and $29.3 \%(77 / 263)$ of the cases, respectively. Double $(n=48)$, triple $(n=21)$, quadruple $(n=4)$, and quintuple $(n=4)$ infections were recorded. Co-infection by $G$. duodenalis and Ancylostoma caninum was the most frequent association identified $(9.4 \% ; 33 / 173)$. The distribution of single and multiple parasite infections according to the status of the investigated dogs is shown in Table 2. Interestingly, urban (pet and categorised as rural animals, whereas pet and breeding dogs were regarded as urban 
two categories: inland (municipalities of Alcalatén, Alto Palancia, and Alto Maestrazgo) and coastal (Bajo Maestrazgo, Plana Alta, and Plana Baja) areas (see also FIG. 1). The male/female ratio was 1.5. Although intestinal parasites were more prevalent in female $(69.1 \% ; 65 / 94)$ than in male $(58.1 \% ; 79 / 136)$ dogs, sex was not a risk factor for infection $(P=0.09)$. As expected, rural dogs were at higher risk of harbouring enteroparasites than urban dogs (PRR: $1.51 ; P<0.001)$. However, no statistically significant differences in the occurrence of enteric pathogens were demonstrated between dogs living in coastal areas and those living in inner regions of the Castellón province (PRR: $1.24 ; P=0.12$ ).

Importantly, surveyed dogs were demonstrated to frequently harbour intestinal protozoan and helminth species potentially infective to humans including G. duodenalis (35.4\%), hookworms (27.0\%), T. canis (8.0\%), Cryptosporidium spp. $(6.8 \%)$, Dipylidium caninum (1.9\%), members of the family Taeniidae (1.5\%), and Blastocystis spp. (1.1\%). Of note, three dogs (1.1\%) were initially found positive for Strongyloides spp. at microscopy. Considering that Castellón has been long regarded as an endemic area for $S$. stercoralis, and that human strongyloidiosis cases are still sporadically detected in the province (Martinez-Perez and Lopez-Velez 2015), we further expanded this preliminary finding. Thus, the presence of Strongyloides spp. was assessed by molecular methods in selected faecal samples $(n=87)$ from dogs considered at higher risk of being infected by the parasite, including shepherd $(n=16)$ and hunting $(n=19)$ dogs. A number of sheltered dogs $(n=52)$ were also tested based on recently published literature (Paradies et al. 2017). Interestingly, PCR-positive results for Strongyloides were obtained in $13.8 \%(12 / 87)$ of the dogs investigated. The infection was more prevalent in shepherd $(25.0 \%, 4 / 16)$ than in sheltered $(15.4 \%, 8 / 52)$ dogs, but was not 
detected in hunting dogs. Neither sex nor origins of the animals were significantly associated to higher Strongyloides spp. infection rates.

\section{Discussion}

This study provides the first description of the diversity and frequency of intestinal parasites in dogs from the Castellón province. The overall canine infection rate $(66 \%)$ recorded here is one of the highest reported in Spain to date, only behind of those $(\sim 70 \%)$ previously identified primarily by microscopy in southern (Martínez-Moreno et al. 2007) and north-eastern Spain (Ortuño et al. 2014). Additionally, a high diversity of intestinal parasites was also identified, including seven protozoa, three cestode, and seven nematode species. Taken together, these data depict an epidemiological scenario characterised by elevated prevalences leading to high infection and (very likely) reinfection rates.

In the European context, our results are in agreement with those documented in Belgium (Claerebout et al. 2009), Czech Republic (Dubná et al. 2007), France (Osman et al. 2015), Germany (Barutzki and Schaper 2003), Greece (Kostopoulou et al. 2017), Italy (Zanzani et al. 2014), and Portugal (Mateus et al. 2014). In these surveys G. duodenalis, $A$. caninum, and $T$. canis were demonstrated to be the most common endoparasite species infecting dogs, although variations in parasite diversity and frequency rates were often reported among different dog populations and geographical areas. Of note, the G. duodenalis infection rates observed in the present study (up to $43.2 \%$ in sheltered dogs), together with that (43.9\%) previously reported in Belgium also by DFAT (Claerebout et al. 2009) are among the highest documented in Europe to date. This fact is probably associated to the superior diagnostic sensitivity of DFAT compared to conventional microscopy, and the high infection pressures and crowded 
conditions commonly seen in kennelled dogs (Gil et al. 2017; Adell-Aledón et al. 2018).

Indeed, sheltered dogs harboured the highest parasite diversity (15 species) detected in the present survey.

Interestingly, shepherd and hunting dogs (both categories linked to rural activities) were significantly more infected by helminth species than dogs from urban areas such as pet and breeding dogs. Thus, infections by hookworms (50-76\%) and $T$. canis (7-17\%) were particularly abundant among the former dog categories. Similar prevalence rates have been previously reported in farm and hunting dogs for A. caninum (70\%) in neighbour Portugal (Mateus et al. 2014), and for T. canis (13\%) in Greece (Papazahariadou et al. 2007). These findings are indicative of failure of dog owners to comply with prescribed deworming protocols.

Data presented here are also relevant from a public veterinary health perspective.

Among the recovered protozoa, G. duodenalis was the most prevalent species. analysed, respectively. Because of their close contact with their owners, these animals may act as potential sources of human giardiosis. In this regard, it should be noted that zoonotic sub-assemblages AII, BIII, and BIV of the parasite have been previously described in sheltered dogs in northern Spain, although the genotypes found seemed primarily transmitted within canine cycles and posed therefore limited risk to humans (Gil et al. 2017). Furthermore, no evidence of zoonotic (or anthroponotic) transmission of $G$. duodenalis was demonstrated between humans and pet dogs sharing households in the geographical area (de Lucio et al. 2017). Similar results and conclusions were reached for the molecular characterization of the G. duodenalis samples generated in the present survey, as described elsewhere (Adell-Aledón et al. 2018). Taken together, all these molecular data indicate that domestic dogs do not play a relevant role as natural 
source of human giardiosis in Spain. Other zoonotic protozoan parasites including Blastocystis spp. and Cryptosporidium spp., were found at lower rates. Toxocara canis represents an important public health concern not only in developing countries but also in industrialized settings with adequate sanitary facilities (Stolk et al. 2016; Salas-Coronas et al. 2018). Over the last few years, toxocariasis has gained an increasing international attention and was listed among the five most neglected parasitic infections according to the US Centers for Disease Control and Prevention (Chen et al. 2018). Human toxocariasis has been described in more than 100 countries, with Spain ranking first among the European countries reporting cases of the visceral form of the disease. Humans acquire the infection via contact with soil contaminated with Toxocara eggs. Toxocara worms have a tendency to cause extraintestinal pathologies including four clinical (visceral larva migrans, ocular toxocariasis, covert toxocariasis, neurotoxocariasis) forms which can lead to serious health consequences. Due to the non-specific symptoms of this disease, its medical and public health impact might be underestimated (Chen et al. 2018). The high prevalence of Toxocara in dogs poses also a considerable public health risk as the eggs are environmentally resistant. Considering that the latest available treatment protocols have improved the control of the disease (Rehbein et al. 2017), it should be emphasized that regular pet deworming would be a useful tool to reduce this problem.

Also noteworthy was the finding of taenid eggs in faecal specimens belonging to sheltered and hunting dogs. The family Taeniidae comprises cestodes of the genus Taenia and Echinococcus, important (and neglected) zoonotic helminths of dogs whose eggs are morphologically indistinguishable at microscopy examination. Although we did not conduct any molecular test for the specific detection of E. granulosus s.l. (the causal agent of human $\mathrm{CE}$ or hydatid disease), the possibility that some of the 
investigated dogs were naturally infected by this cestode cannot be completely ruled out. Indeed, an E. granulosus infection rate of $0.5 \%(5 / 1,040)$ by necropsy has been previously described in sheltered dogs in Northern Spain (Benito et al. 2003).

Therefore, more studies are required to investigate the current epidemiological situation of canine equinococcosis in this geographical area. One of the most intriguing contributions of this paper was the detection of Strongyloides spp. in a significant number of shepherd and sheltered (but not hunting) dogs. Members of the family Canidae and Felidae are considered suitable hosts for a number of Strongyloides species including S. stercoralis, the etiological agent of human strongyloidiosis (Thamsborg et al. 2017). Whether domestic dogs can act as suitable reservoirs of human infections remains a matter of intense debate, but a recent molecular survey conducted in rural Cambodia has demonstrated that humans and their dogs can be infected by the same genetic variant of S. stercoralis (Jaleta et al. 2017). Arguing in favour of the occurrence of zoonotic transmission, the authors suggested that in order to reduce the exposure of humans to infective $S$. stercoralis larvae, dogs should be treated against the infection along with their owner. In Europe there are few studies on the prevalence of this parasite in dogs. The infected animals were usually asymptomatic and when signs and symptoms appeared they were unspecific. However, the increase of human strongyloidiasis cases diagnosed globally has lead the scientific community to reconsider the role of domestic dogs as potential natural reservoirs of human infections (Paradies et al. 2017). Imported human strongyloidiasis associated to immigrant populations and returning travellers from endemic areas is increasingly reported in Spain (Martinez-Perez et al. 2018, Belhassen-García et al. 2017), although in Castellón Province sporadic autochthonous cases of the disease are still recorded. The fact that these cases correspond to individuals of older age has been interpreted as evidence of 
successful interruption of the transmission cycle of the parasite (Martinez-Perez and Lopez-Velez, 2015). Still, it would be very interesting to isolate Strongyloides larvae from fresh faecal material of canine origin in order to identify the species involved and assess the associated zoonotic risk.

\section{Conclusions}

This is the first coprological, microscopy-based study targeting different dog populations conducted to date in the Castellón Province. Investigated dogs were infected at high rates by a wide range of protozoa and helminth species, some of them with zoonotic potential. Dogs from rural areas (mainly shepherd and hunting dogs) were more exposed. Simple measures, such as periodic deworming, prompt removal of faeces from kennels, and improving owner's education on zoonotic transmission are all costeffective methods to limit the risk of animal and human infections by enteric parasites. People at higher risk of infection (e.g. veterinarians, slaughterhouse workers, animal husbandry workers, kennel personnel, and hunters) should be provided with accurate information on the potential risks associated to dog handling and management. Finally, against these infections.

\section{Acknowledgements}

This study was funded by grants from CEU-Santander Program under project Zoocan Study, Universidad CEU Cardenal Herrera, Spain. The authors are grateful to Sandra Diago Porcar for her technical assistance during sampling and methodological procedures. 


\section{Author Disclosure Statement}

349 No competing financial interests exist.

350

351

352

\section{References}

Adell-Aledón M, Köster PC, de Lucio A, Puente P, et al. Occurrence and molecular epidemiology of Giardia duodenalis infection in dog populations in Eastern Spain. BMC Vet Res 2018;14:1-11.

Barutzki D, Schaper R. Endoparasites in dogs and cats in Germany 1999-2002. Parasitol Res 2003;90 (Suppl 3):148-150.

Benito A, Carmena D, Postigo I, Estíbalez JJ, et al. Intestinal helminths in dogs in Alava, North of Spain. Res Rev Parasitol 2003;63:121-126.

Belhassen-García M, Alonso-Sardón M, Martinez-Perez A, Soler C, et al. Surveillance of strongyloidiasis in Spanish in-patients (1998-2014). PLoS One 2017;28:e189449.

Causapé AC, Quílez J, Sánchez-Acedo C, del Cacho E. Prevalence of intestinal parasites, including Cryptosporidium parvum, in dogs in Zaragoza city, Spain. Vet Parasitol 1996;67:161-167.

Claerebout E, Casaert S, Dalemans AC, De Wilde N, et al. Giardia and other intestinal parasites in different dog populations in Northern Belgium. Vet Parasitol 2009;161:41-46.

Chen J, Liu Q, Liu GH, Zheng WB, et al. Toxocariasis: a silent threat with a progressive public health impact. Infect Dis Poverty 2018;7(1):59.

Dantas-Torres F, Otranto D. Dogs, cats, parasites, and humans in Brazil: opening the black box. Parasit Vectors 2014;7:1-25. 
de Lucio A, Bailo B, Aguilera M, Cardona GA, et al. No molecular epidemiological evidence supporting household transmission of zoonotic Giardia duodenalis and Cryptosporidium spp. from pet dogs and cats in the province of Álava, Northern Spain. Acta Trop 2017; 170:48-56.

Dean AG, Sullivan KM, Soe MM. OpenEpi: Open source epidemiologic statistics for public health, Version 3.01. www.OpenEpi.com. (Accessed 18 February 2019).

Deplazes P, van Knapen F, Schweiger A, Overgaauw PA. Role of pet dogs and cats in the transmission of helminthic zoonoses in Europe, with a focus on echinococcosis and toxocarosis. Vet Parasitol 2011;182:1-53.

Dubná S, Langrová I, Nápravník J, Jankovská I, et al. The prevalence of intestinal parasites in dogs from Prague, rural areas, and shelters of the Czech Republic. Vet Parasitol 2007;145:120-128.

Gil H, Cano L, de Lucio A, Bailo B, et al. Detection and molecular diversity of Giardia duodenalis and Cryptosporidium spp. in sheltered dogs and cats in Northern Spain. Infect Genet Evol 2017;50:62-69.

Gracenea M, Gómez MS, Torres J. Prevalence of intestinal parasites in shelter dogs and cats in the metropolitan area of Barcelona (Spain). Acta Parasitologica 2009;54:73-77.

Jaleta TG, Zhou S, Bemm FM, Schär F, et al. Different but overlapping populations of Strongyloides stercoralis in dogs and humans-Dogs as a possible source for zoonotic strongyloidiasis. PLoS Neglect Trop Dis 2017;11:1-21.

Kostopoulou D, Claerebout E, Arvanitis D, Ligda P, et al. Abundance, zoonotic potential and risk factors of intestinal parasitism amongst dog and cat populations: The scenario of Crete, Greece. Parasit Vectors 2017;10:43. 
Martínez-Carrasco C, Berriatua E, Garijo M, Martínez J, et al. Epidemiological study of non-systemic parasitism in dogs in southeast Mediterranean Spain assessed by coprological and post-mortem examination. Zoonoses Public Health 2007;54:195203.

Martínez-Moreno FJ, Hernández S, López-Cobos E, Becerra C, et al. Estimation of canine intestinal parasites in Córdoba (Spain) and their risk to public health. Vet Parasitol 2007;143:7-13.

Martinez-Perez A, Lopez-Velez R. Is strongyloidiasis endemic in Spain? PLoS Neglect Trop Dis 2015;9:e0003482.

Martinez-Perez A, Roure Díez S, Belhassen-Garcia M, Torrús-Tendero D, et al. Management of severe strongyloidiasis attended at reference centers in Spain PLoS Neglect Trop Dis 2018;23:e006272.

Mateus TL, Castro A, Ribeiro JN, Vieira-Pinto M. Multiple zoonotic parasites identified in dog feces collected in Ponte de Lima, Portugal-a potential threat to human health. Int Jf Environ Res Public Health 2014;11:9050-9067.

Miró G, Mateo M, Montoya A, Vela E, et al. Survey of intestinal parasites in stray dogs in the Madrid area and comparison of the efficacy of three anthelmintics in naturally infected dogs. Parasitol Res 2007;100:317-320.

Ortuño A, Scorza V, Castellà J, Lappin M. Prevalence of intestinal parasites in shelter and hunting dogs in Catalonia, Northeastern Spain. Vet J 2014;199:465-467.

Otranto D, Dantas-Torres F, Mihalca AD, Traub RJ, et al. Zoonotic parasites of sheltered and stray dogs in the era of the global economic and political crisis. Trends Parasitol 2017;33:813-825.

Overgaauw PA, van Knapen F. Veterinary and public health aspects of Toxocara spp. Vet Parasitol 2013;193:398-403. 
Papazahariadou M, Founta A, Papadopoulos E, Chliounakis S, et al. Gastrointestinal parasites of shepherd and hunting dogs in the Serres Prefecture, Northern Greece. Vet Parasitol 2007;148:170-173.

Paradies P, Iarussi F, Sasanelli M, Capogna A, et al. Occurrence of strongyloidiasis in privately owned and sheltered dogs: clinical presentation and treatment outcome. Parasit Vectors 2017;10:345.

Rehbein S, Knaus M, Mallouk Y, Breiltgens T, et al. Efficacy against nematode infections and safety of afoxolaner plus milbemycin oxime chewable tablets in domestic dogs under field conditions in Europe. Parasitol Res 2017;116:259-269.

Registro Informático Valenciano de Identificación Animal. http://www.rivia.org/. (accessed 18 February 2019).

Salas-Coronas J, Cabezas-Fernández MT, Lozano-Serrano AB, Soriano-Pérez MJ, et al. Newly arrived African migrants to Spain: Epidemiology and burden of disease. Am J Trop Med Hyg 2018;98:319-325.

Saugar JM, Merino FJ, Martín-Rabadán P, Fernández-Soto P, et al. Application of realtime PCR for the detection of Strongyloides spp. in clinical samples in a reference center in Spain. Acta Trop 2015;42:20-25.

Soriano SV, Pierangeli NB, Roccia I, Bergagna HF et al. A wide diversity of zoonotic intestinal parasites infects urban and rural dogs in Neuquén, Patagonia, Argentina. Vet Parasitol 2010;167:81-85.

Stolk WA, Kulik MC, le Rutte EA, Jacobson J et al. Between-country inequalities in the neglected tropical disease burden in 1990 and 2010, with projections for 2020. PLoS Neglect Trop Dis 2016;10:e004560.

Thamsborg SM, Ketzis J, Horii Y, Matthews JB. Strongyloides spp. infections of veterinary importance. Parasitology 2017;144:274-284. 

Coprologique. In: Beerse, Belgique, ed. Belgium: Janssen Research Foundation; 1979:35-36.

449

450

451

452

453

454

455

456

457

458

459

460

461

462

463

464

465

466

467

468

469

470

Verweij JJ, Canales M, Polman K, Ziem J, et al. Molecular diagnosis of Strongyloides stercoralis in faecal samples using real- time PCR. Trans R Soc Trop Med Hyg 2009;103:342-346.

Zanzani SA, Gazzonis AL, Scarpa P, Berrilli F, et al. Intestinal parasites of owned dogs and cats from metropolitan and micropolitan areas: prevalence, zoonotic risks, and pet owner awareness in northern Italy. BioMed Res Int 2014;2014:696508.

Address correspondence to:

Paula Sanchez

Department of Medicine, Cardenal Herrera-CEU University, CEU Universities, C/

Grecia, 31, 12006 Castellón de la Plana, Castellón, Spain.

E-mail: paula.sanchez@uchceu.es

Address correspondence to:

David Carmena

Parasitology Reference and Research Laboratory, National Centre for Microbiology, Health Institute Carlos III. Ctra. Majadahonda-Pozuelo Km 2, 28220 Majadahonda, Madrid, Spain.

E-mail: dacarmena@,isciii.es

\section{Figure legends}

FIG.1. Map of the administrative divisions of the Castellón province. The municipalities where sampling was conducted and the status of the dog sub-populations 
471 are indicated. The location of Castellón in Spain is highlighted in red in the upper left 472 corner. Image reproduced with permission of BioMed Central.

473 